CVH trajectory from middle age to older age and subsequent HF risk.

Methods In 1998-2000, 20 years after the initial screening of 7735 men aged 40-59 years (The British Regional Heart Study), 4252 men were re-examined when aged 60-79 years, with collection of all LS7 components and follow-up for a median period of 15.8 years. Men with previous history of cardiovascular disease were excluded leaving 3698 men. The composite LS7 score ranges from $0-14$ and men were classified as having poor (1-4), intermediate (5-10) and ideal (1114) $\mathrm{CVH}$ score. Four CVH trajectory groups were created based on transition between low and high $\mathrm{CVH}$ score from middle to older age:(1) Low-Low, (2) Low-High, (3), HighLow, and (4) High-High. Cox models were used to estimate the risk of HF adjusted for age, alcohol consumption, socioeconomic class and incident myocardial infarction.

Results Of the 3698 men 14\% $(n=522)$ had ideal CVH score. Ideal $\mathrm{CVH}$ was associated with a significant decrease in risk of $\mathrm{HF}$ compared to those with a poor $\mathrm{CVH}$ score (HR 0.52, $95 \% 0.31$ to $0.88, \mathrm{p}<0.016)$; intermediate $\mathrm{CVH}$ was associated with reduced but non-significant $\mathrm{HF}$ risk (HR 0.79, 95\% CI 0.51 to $1.24, \mathrm{p}=0.310$ ). Compared to the Low-Low CVH trajectory group, those who maintained a healthy $\mathrm{CVH}$ score (High-High) showed the lowest risk of HF (HR 0.67, 95\% CI 0.51 to $0.87, \mathrm{p}<0.003)$; those who moved from high to low showed lower but non-significant risk (HR 0.78 95\% CI 0.55 to $1.11, \mathrm{p}=0.17)$; those who moved from low to high showed no benefit (HR $1.0195 \%$ CI 0.76 to 1.33).

Discussion Our findings suggest that having ideal $\mathrm{CVH}$ reduces the risk of developing HF in older age. LS7 is a simple way to identify high risk individuals however the prevalence of older men with ideal $\mathrm{CVH}$ is low. Adopting and maintaining healthy cardiovascular health from middle age to older age confers the most benefit in preventing $\mathrm{HF}$ in later life.

\section{OP08 SOCIOECONOMIC POSITION AND SEX-SPECIFIC TRAJECTORIES OF METABOLITES FROM CHILDHOOD TO EARLY ADULTHOOD: A PROSPECTIVE COHORT STUDY}

${ }^{1}$ Kate O'Neill*, 1,2,3 Linda O'Keeffe. 'School of Public Health, University College Cork, Cork, Ireland; ${ }^{2}$ MRC Integrative Epidemiology Unit, University of Bristol, Bristol, UK; ${ }^{3}$ Population Health Sciences, Bristol Medical School, Bristol, UK

\subsection{6/jech-2021-SSMabstracts.8}

Background Socioeconomic inequalities in cardiovascular disease are stronger and more consistent in females compared with males. However, mechanisms underlying these inequalities and whether they emerge in early life are unclear.

Methods Trajectories of 148 metabolic trait concentrations from age $7 \mathrm{y}$ to $25 \mathrm{y}$ in a contemporary English birth cohort, The Avon Longitudinal Study of Parents and Children (ALSPAC), were analysed. Outcomes included concentrations of metabolic traits quantified using nuclear magnetic resonance spectroscopy measured at 7y,15y, 18y and 25y. Maternal education was used as an indicator of socioeconomic position (SEP), reported by mothers at 32-weeks gestation. Using linear spline multilevel models, sex-specific associations of SEP and trajectories of each metabolic trait concentration were examined. Sex-specific associations were converted to standard deviation (SD) units by dividing the predicted total absolute difference from $7 \mathrm{y}$ to $25 \mathrm{y}$ by SEP in original units by the sex-specific SD of the trait in the reference SEP category (degree level maternal education).

Results Total participants included ranged from 5,980-6,212 with 10,023-11,945 repeated measures. SEP was associated with numerous metabolic traits trajectories in females, some which developed or strengthened by age $25 \mathrm{y}$ with evidence of an emerging SEP gradient. Associations were strongest for large HDL and VLDL cholesterol, apolipoprotein B/A-1 and glycoprotein acetyls. For instance, by age 25y less than O-level education was associated with 0.37 SD (95\% CI: 0.21, 0.5.3), O-level with 0.28 SD (95\% CI: 0.14, 0.42) and A-level with 0.08 SD (95\% CI: -0.6, 0.22) higher concentrations of glycoprotein acetyls compared to degree level education. In males, associations between SEP and metabolic traits were weaker and less consistent with little evidence of an SEP gradient.

Conclusion Sex differences in socioeconomic inequalities in cardiometabolic risk appear to develop early in the life course and are evident by early adulthood with more adverse effects of SEP in females.

\section{OP09 QUANTIFYING BENEFITS OF THE DANISH TRANSFAT BAN FOR CORONARY HEART DISEASE MORTALITY 1991-2007: SOCIOECONOMIC ANALYSIS USING THE IMPACT $_{S E C}$ MODEL}

${ }^{1}$ Kirsten Schroll Bjoernsbo*, ${ }^{2}$ Albert Marni Joense, ${ }^{1,3,4}$ Torben Joergensen, ${ }^{5}$ Søren LundbyeChristensen, ${ }^{6}$ Anette Bysted, ${ }^{6}$ Tue Christensen, ${ }^{7}$ Simon Capewell, ${ }^{7}$ Martin O'Flaherty. ${ }^{1}$ Center for Clinical Research and Prevention, Bispebjerg and Frederiksberg Hospital, Frederiksberg, Denmark; ${ }^{2}$ Aalborg University Hospital, Department of Cardiology, Aalborg, Denmark; ${ }^{3}$ Department of Public Health, Faculty of Health and Medical Sciences, University of Copenhagen, Copenhagen Denmark; ${ }^{4}$ Faculty of Medicine, Aalborg University, Aalborg, Denmark; ${ }^{5}$ Unit of Clinical Biostatistics, Aalborg University Hospital, Aalborg, Denmark; ${ }^{6}$ National Food Institute, Technical University of Denmark, Kgs. Lyngby, Denmark; ${ }^{7}$ University of Liverpool, Department of Public Health and Policy, Liverpool, UK

\subsection{6/jech-2021-SSMabstracts.9}

Background In 2004, the Danish Transfat Ban was implemented (Order no. 160, 2003). To determine health and equity benefits of this regulation on industrially produced trans fatty acid (ITFA) content in Danish food, we quantified the changes in ITFA intake from 1991 to 2007. We then estimated the relative contributions of changes in ITFA consumption, other cardiovascular risk factors and treatments on Coronary Heart Disease (CHD) mortality, all stratified by socioeconomic group.

Methods Participants included adult Danes aged 25-84 years living in Denmark in 1991 and 2007, stratified by socioeconomic quintiles. Population counts were obtained from the Danish Central Office of Civil Registration, and financial income data from Statistics Denmark. ITFA intake data as grams/day and $\%$ total energy intake $(\% \mathrm{E})$ were obtained from dietary assessments from the DanMonica 3 Study (1991) and the Danish National Surveys of Dietary Habits and Physical Activity (DANSDA, 2005-2007) combined with estimated ITFA content in foods on the Danish market. We extended the previously validated Danish IMPACT $_{\mathrm{SEC}}$ model to quantify reductions in CHD mortality; the principal output being the number of CHD deaths prevented or postponed attributable to changes in ITFA (\%E) intake between 1991 - 2007, all stratified by socioeconomic group. To quantify degrees of uncertainty, we conducted robust sensitivity analyses.

Results Between 1991 and 2007, mean ITFA intake in Denmark fell dramatically from $2.9 \mathrm{~g} / \mathrm{d}$ to $0.3 \mathrm{~g} / \mathrm{d}$ in men, and 